\title{
Sensitivity of the r-process to nuclear masses
}

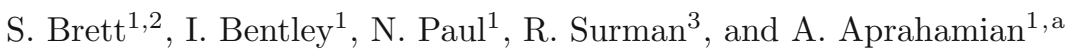 \\ 1 Dept. of Physics, University of Notre Dame, Notre Dame, IN 46556, USA \\ 2 Dept. of Physics, University of Surrey, Guildford, Surrey, GU2 7XH, UK \\ ${ }^{3}$ Dept. of Physics, Union College, Schenectady, NY 12308, USA
}

Received: 23 October 2012

Published online: 12 December 2012

(c) The Author(s) 2012. This article is published with open access at Springerlink.com Communicated by J. Wambach

\begin{abstract}
The rapid neutron capture process (r-process) is thought to be responsible for the creation of more than half of all elements beyond iron. The scientific challenges to understanding the origin of the heavy elements beyond iron lie in both the uncertainties associated with astrophysical conditions that are needed to allow an r-process to occur and a vast lack of knowledge about the properties of nuclei far from stability. There is great global competition to access and measure the most exotic nuclei that existing facilities can reach, while simultaneously building new, more powerful accelerators to make even more exotic nuclei. This work is an attempt to determine the most crucial nuclear masses to measure using an r-process simulation code and several mass models (FRDM, Duflo-Zuker, and HFB-21). The most important nuclear masses to measure are determined by the changes in the resulting r-process abundances. Nuclei around the closed shells near $N=50,82$, and 126 have the largest impact on r-process abundances irrespective of the mass models used.
\end{abstract}

Basic properties of nuclei, such as their binding energies per nucleon allow the synthesis of the elements up to approximately iron via fusion reactions in stars from the lightest elements created by the Big Bang. However, the abundances of elements in our solar system contain a substantial number of nuclei well beyond iron [1-3]. The origins of these nuclei are entangled in complexity since the heavier elements are thought to be made via both slow and rapid neutron-capture processes ( $\mathrm{s}$ - and r-processes) [4]. The s-process leads to a network of nuclei near stability while the r-process allows the production of nuclei with increasing neutron numbers much further from stability, producing neutron-rich nuclei. The astrophysical scenarios in which the s-process can take place have been identified, but a potential site for the r-process is still unresolved [5]. The challenge for astrophysical science today is to understand the conditions that would provide a major abundance of neutrons and lead to successive captures before the nucleus has a chance to decay; while on the nuclear side, the challenge is to determine the physics of nuclei far from stability where the range and impact of the nuclear force is less well known $[6,5]$.

There have been a number of astrophysical scenarios suggested as possible sites for the r-process. Some

\footnotetext{
a e-mail: aapraham@nd.edu
}

of the most promising sites include the neutrino-driven wind from core-collapse supernovae [7], two-neutron star mergers [8], gamma-ray bursts [9], black-hole neutron star mergers [10], relativistic jets associated with failed supernovae [11] or magnetohydrodynamic jets from supernovae $[12]$.

The r-process proceeds via a sequence of neutron captures, photodissociations and $\beta$-decays. Simulations of the $r$-process therefore require tabulations of $\beta$-decay lifetimes, neutron capture rates and neutron separation energies; photodissociation rates are determined from the capture rates and separation energies by detailed balance [13],

$$
\lambda_{\gamma}(Z, A) \propto T^{3 / 2} \exp \left[-\frac{S_{n}(Z, A)}{k T}\right]\langle\sigma v\rangle_{(Z, A-1)} .
$$

In the above expression, $T$ is the temperature, $\langle\sigma v\rangle_{(Z, A-1)}$ is the thermally averaged value of the neutron capture cross-section for the neighboring nucleus with one less neutron, and $S_{n}(Z, A)$ is the neutron separation energy - the difference in binding between the nuclei $(Z, A)$ and $(Z, A-1)$. Nuclear masses are crucial inputs in theoretical calculations of each of these sets of nuclear data.

One way to assess the role of nuclear masses in the r-process is to choose two or more mass models, calculate all of the relevant nuclear data with the mass model consistently, and then run r-process simulations with the 
different sets of global data. Such comparisons are quite valuable and examples include refs. [14-16]. Our approach here is quite different. We instead focus on the sensitivity of the r-process to the individual neutron separation energies within a given mass model, as they appear in eq. (1), in an attempt to determine the nuclei that have the greatest impact on the overall r-process abundances and, in turn, identify the most crucial measurements to be made. This is the first time that such an attempt has been made and the results could potentially be of great significance to both nuclear and astrophysical science.

The study of radioactive nuclei far from stability approaching the r-process path is one of the global research frontiers for nuclear science today. New facilities are being developed in the USA (CARIBU at ANL, NSCL and FRIB at MSU), in Europe (ISOLDE at CERN), in France (SPIRAL II at GANIL), in Finland (Jyvaskyla), in Germany (FAIR at GSI Darmstadt), in Japan (RIKEN), in China (BRIF, CARIF in CIAE Beijing), and in Canada (ISAC at TRIUMF). The overarching question for this global effort in nuclear science is which measurements need to be made [17].

This study used a fully dynamical r-process nuclear network code [18]. Inputs to the simulation code include a seed nucleus, neutron density, temperature and dynamical timescale descriptive of a given astrophysical scenario. In addition, $\beta$ decay rates, neutron capture rates and neutron separation energies are the inputs for the nuclear properties. The simulation processes neutron captures, photodissociations, $\beta$-decays, and $\beta$-delayed neutron emissions from the start of the r-process through freezeout and the subsequent decay toward stability [19]. Fission, while important in some astrophysical scenarios, is not significant for the the conditions used here and so is not included.

All the calculations are done for the same initial astrophysical conditions. The astrophysical scenario used in our simulations was based on the $\mathrm{H}$ or high-frequency r-process suggested by Qian et al. [20], with an initial temperature of $T_{9}=1.5$ and an initial density of $3.4 \times 10^{2} \mathrm{~g} / \mathrm{cm}^{3}$. We take the temperature and density to decline exponentially as in [21] with a dynamical timescale of $0.86 \mathrm{~s}$. While Qian specifies a seed of ${ }^{90} \mathrm{Se}$ and a neutron to seed ratio $\left(N_{n} / N_{\text {seed }}\right)$ of 86 [20], here a lighter seed of ${ }^{70} \mathrm{Fe}$ is chosen, which results in $N_{n} / N_{\text {seed }}=67$ when the electron fraction is kept consistent with Qian $\left(Y_{e}=0.190\right)$.

The nuclear data inputs include beta-decay rates from [22] and neutron capture rates from [23], both calculated with Finite Range Droplet Model (FRDM) masses. The measured values of $S_{n}$ come from the Audi Mass Evaluation 2003 [24]. For the remaining nuclei, we used the $S_{n}$ values resulting from the calculated mass values in the FRDM [25]. We subsequently varied these theoretical $S_{n}$ for one nucleus at a time by $\pm 25 \%$. In each case, the resulting r-process abundance curves were generated and compared against the baseline abundances resulting from the unchanged $S_{n}$ value.

The $25 \%$ variation of separation energies was chosen somewhat arbitrarily. A comparison of the ratio of separation energies extracted from measured masses or the-

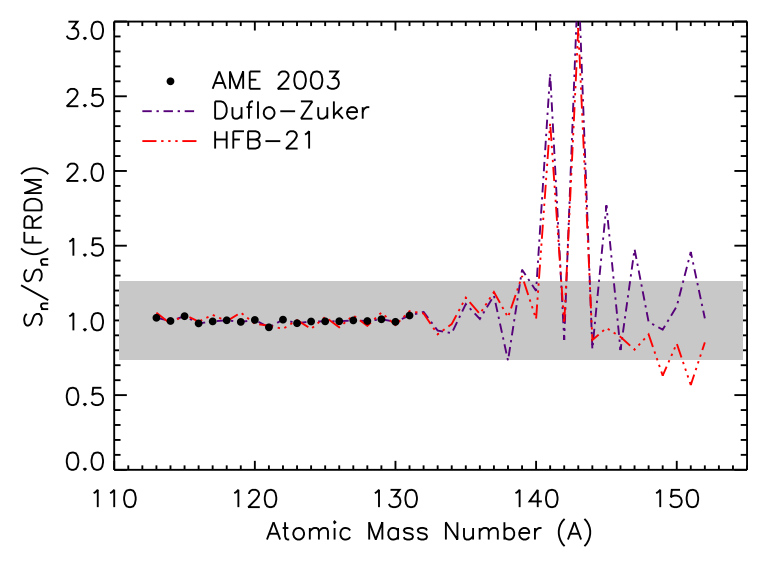

Fig. 1. Comparison of the separation energies from DufloZuker [26], HFB-21 [27], and the experimental masses from [24] to the FRDM [25] values for the tin isotopes.

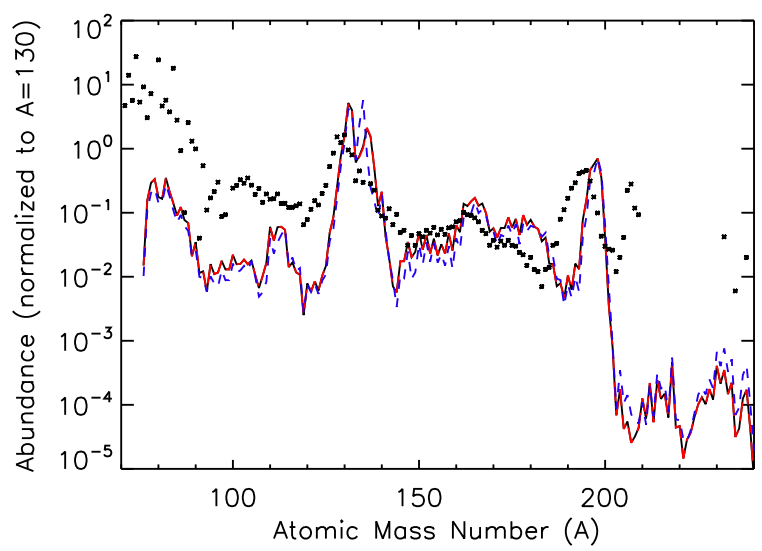

Fig. 2. Final r-process abundances for the baseline Hscenario [20] with ${ }^{70} \mathrm{Fe}$ seed (black line) compared to simulations in which the neutron separation energy of ${ }^{138} \mathrm{Sn}$ is increased (red long-dashed line) or decreased (blue short-dashed line) by $25 \%$. The calculated abundances are normalized to the solar r-process abundances of Sneden et al. [4] (points) at $A=130$

oretically calculated separation energies with the FRDM calculated values is shown for the Sn isotopes in fig. 1 . This indicates that the $25 \%$ value is a reasonable variation estimate far from stability.

An example of the resulting abundance patterns is shown in fig. 2, where the baseline pattern is compared to the final abundance patterns produced by simulations in which the separation energy of ${ }^{138} \mathrm{Sn}$ was increased or decreased by $25 \%$. This comparison can be quantified by summing the differences in the final mass fractions,

$$
\left.F_{ \pm}=100 \sum_{A} \mid X_{\text {baseline }}(A)-X_{ \pm \Delta S_{n}}(A)\right) \mid
$$

where $X(A)=A Y(A)$ is the mass fraction of nuclei with mass number $A$ (such that $\sum_{A} X(A)=1$ ), and the sum of 


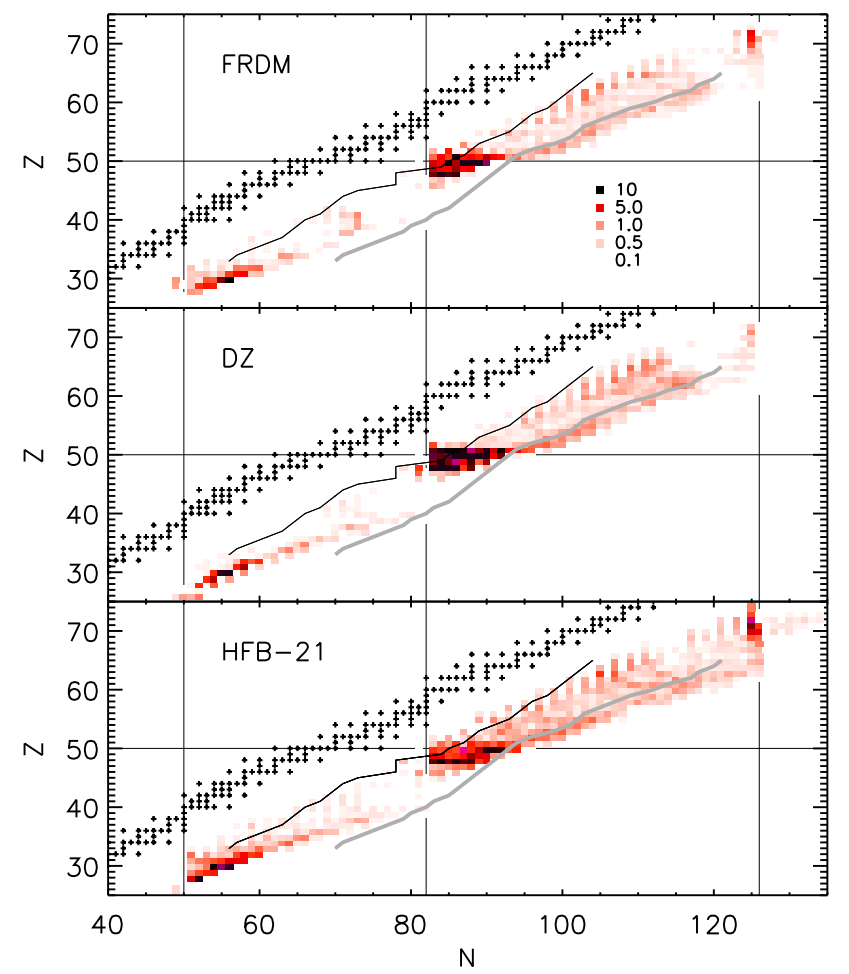

Fig. 3. Comparison of the sensitivity to mass values determined by eq. (2). The separation energies far from stability were generated by the FRDM [25], Duflo-Zuker [26], and HFB-21 [27]. The scale is from white to dark red, indicating regions with a small change to a substantial change in the resulting abundances. For reference, stable nuclei have been included as black crosses and the magic numbers have been indicated by thin lines. Superimposed on the sensitivity results are the limits of accessibility by CARIBU [28] and the proposed FRIB intensities [29]. In both cases, we have plotted the conservative limits of what can be produced and measured in mass measurements.

$A$ ranges over the entire abundance curve. This quantity is largest when the curves differ near the peak abundances, giving preference to those regions.

The values of $F=\left(F_{+}+F_{-}\right) / 2$ are calculated for 3010 nuclei from ${ }^{58} \mathrm{Fe}$ to ${ }^{294} \mathrm{Fm}$. Figure 3 shows the nuclei whose separation energy variations result in the greatest changes in the resulting r-process abundances. Nuclei that have the greatest impact on the r-process are those neutron rich nuclei near the closed shells at $Z=28$ and 50, and $N=50,82$, and 126 .

A natural question to ask is the dependence of these results on the mass model used. Therefore, similar calculations were performed using four additional mass models, the Duflo-Zuker (DZ) [26], the Extended Thomas Fermi plus Strutinsky Integral with shell Quenching (ETFSIQ) [30], the Hartree-Fock-Bogoliubov (HFB-21) [27], and the F-spin [31] model in addition to the FRDM. All models take advantage of very different physics ingredients to calculate the masses of nuclei far from stability. Each of the calculations performed started with the same initial astrophysical conditions and again varying individual sep- aration energies by $\pm 25 \%$. The results are astounding. In each case, the nuclei with the greatest impact were generally the ones near the major closed shells independent of the chosen mass models. Figure 3 shows the resulting sensitivity plots from three of the mass models; the FRDM, DZ, and HFB-21 models. Nuclei near the closed shells of $N=50,82$, and 126 rise above all the others in impact. The nuclei with the most impact on the r-process abundances cluster around ${ }^{132} \mathrm{Cd}$ and ${ }^{138} \mathrm{Sn}$. In this region, the nuclei are ${ }^{131-134} \mathrm{Cd},{ }^{132-137} \mathrm{In},{ }^{135-140} \mathrm{Sn},{ }^{139,141} \mathrm{Sb}$. There are also specific low mass nuclei such as ${ }^{82} \mathrm{Cu},{ }^{85} \mathrm{Zn}$, and ${ }^{88} \mathrm{Zn}$ that are important.

In trying to understand these results, we know that there are two ways that an individual neutron separation energy can influence the r-process abundance distribution. The first is a long-recognized [32] equilibrium effect, and the second is an early freezeout photodissociation effect, recently pointed out in [33]. In the classic view, the rprocess takes place in conditions of $(n, \gamma)-(\gamma, n)$ equilibrium, where abundances along an isotopic chain are determined by a Saha equation,

$$
\begin{aligned}
I_{00}= & \frac{Y(Z, A+1)}{Y(Z, A)}=\frac{G(Z, A+1)}{2 G(Z, A)}\left(\frac{2 \pi \hbar^{2} N_{A}}{m_{n} k T}\right)^{3 / 2} N_{n} \\
& \times \exp \left[\frac{S_{n}(Z, A+1)}{k T}\right],
\end{aligned}
$$

where the $G$ s are the partition functions, $N_{n}$ is the neutron number density, and $m_{n}$ is the nucleon mass. The relative abundances of the different isotopic chains are then determined by the $\beta$-decay lifetimes of the most populated nuclei along each chain. As described in eq. (3), any change to an individual separation energy will cause a shift in the abundances along the isotopic chain. This can have a global impact on the final abundance pattern, particularly if the affected nucleus is highly populated and material is shifted to a nucleus with a significantly faster or slower $\beta$ decay lifetime. For example, consider the case of ${ }^{138} \mathrm{Sn}$, a nucleus just above the $N=82$ closed shell region. In the baseline simulation, ${ }^{138} \mathrm{Sn}$ is the most abundant tin isotope, and ${ }^{136} \mathrm{Sn}{ }^{140} \mathrm{Sn}$ are much less abundant. Their mass fractions are shown as a function of time in fig. 4(a); their relative values follow those predicted by eq. (3) until about $t \sim 1.2 \mathrm{~s}$, when equilibrium begins to fail and the nuclei primarily $\beta$-decay to stability. If the simulation is repeated with neutron separation energy of ${ }^{138} \mathrm{Sn}$ reduced by $25 \%$, we see that the equilibrium abundance of this nucleus is drastically reduced, as expected from eq. (3) and shown in fig. 4(b). Material is instead shifted to ${ }^{136} \mathrm{Sn}$, which has a $\beta$-decay lifetime approximately 1.6 times that of ${ }^{138} \mathrm{Sn}$ (and 5.3 times the lifetime of ${ }^{140} \mathrm{Sn}$, which is also depleted by the shift). As a result, more material is stuck in the tin isotopic chain compared to the baseline simulation, and the overall rate at which neutrons are consumed is slowed, as shown in fig. 4(c). This impacts the availability of neutrons for the whole abundance pattern and results in changes throughout the pattern. The second mechanism, in contrast, operates once $(n, \gamma)-(\gamma, n)$ equilibrium begins to fail, and individual neutron capture and photodissociation rates become important. Since the neutron separation 


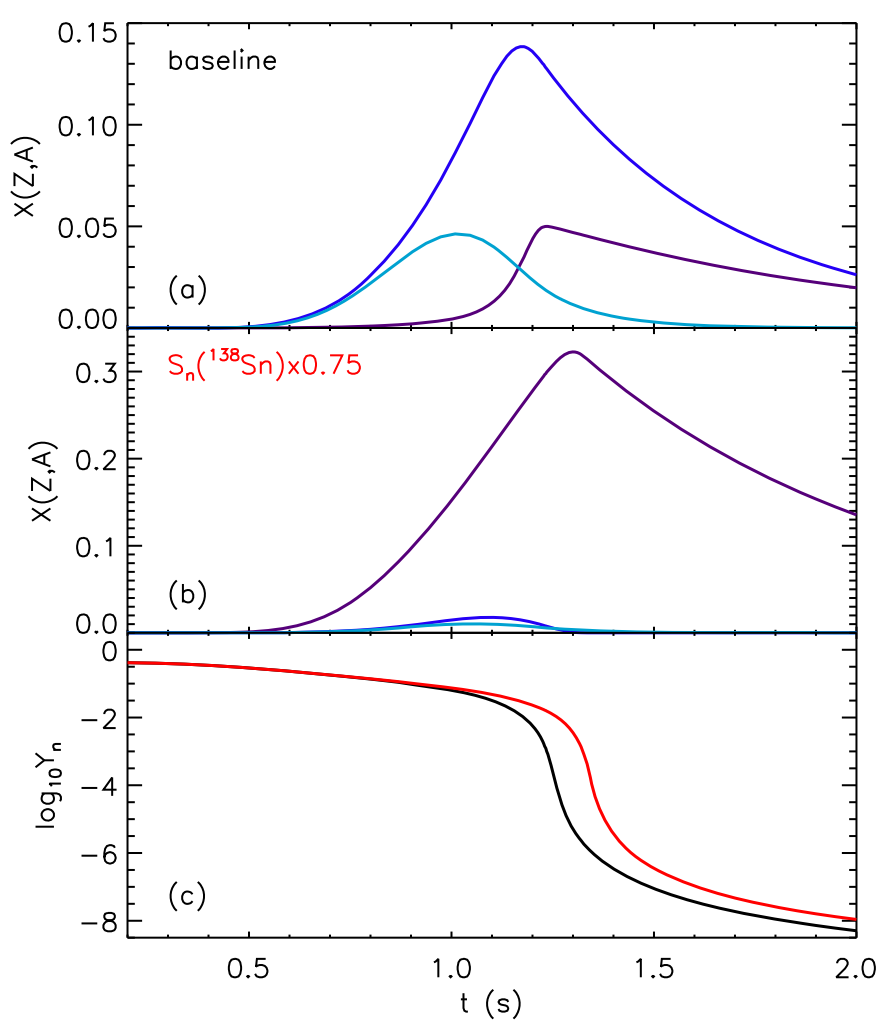

Fig. 4. Shows the mass fractions of ${ }^{136} \mathrm{Sn}$ (purple), ${ }^{138} \mathrm{Sn}$ (blue), and ${ }^{140} \mathrm{Sn}$ (aqua) for the baseline r-process simulation (top panel) and the simulation with the separation energy of ${ }^{138} \mathrm{Sn}$ decreased by $25 \%$ (middle panel). The bottom panel compares the neutron abundance for the two simulations (black and red lines, respectively).

energy appears in the exponential in eq. (1), photodissociation rates are quite sensitive to this quantity. Changes in individual photodissociation rates during freezeout can produce local shifts in abundances, which can translate into global abundance changes if they alter the late-time availability of free neutrons. This mechanism is described carefully in [33]. Odd- $N$ nuclei, which tend to be in equilibrium only briefly if at all, are particularly susceptible to these non-equilibrium effects.

In conclusion, this study of 3010 nuclei via an r-process simulation tested the sensitivity of the r-process abundance yields to the theoretical mass values of neutron rich nuclei presently unknown in the laboratory from several different mass models, the results are shown here for three of them (FRDM [25], Duflo-Zuker [26], and HFB-21 [27]). The results are uniform and conclusive in highlighting the importance of nuclei near closed shells. Essentially the same set of nuclei emerge as having the highest impact on the r-process irrespective of the varying physics ingredients of the different mass models. The nuclei with greatest impact on the r-process - neutron-rich isotopes of cadmium, indium, tin, and antimony in the $N=82$ region, nickel, copper, zinc, and gallium in the $N=50$ region, and thulium, ytterbium, lutetium, and hafnium in the $N=126$ region - should be of highest priority to measure in the various exotic beam facilities around the
Table 1. Most important neutron separation energies for the $\mathrm{H}$-scenario with ${ }^{70} \mathrm{Fe}$ seed.

\begin{tabular}{cccccc}
\hline \multicolumn{3}{c}{ FRDM } & \multicolumn{3}{c}{$\mathrm{DZ}$} \\
\hline${ }^{A} \mathrm{X}$ & $F$ & ${ }^{A} \mathrm{X}$ & $F$ & ${ }^{A} \mathrm{X}$ & $F$ \\
${ }^{138} \mathrm{Sn}$ & 24.59 & ${ }^{132} \mathrm{Cd}$ & 36.54 & ${ }^{140} \mathrm{Sn}$ & 17.59 \\
${ }^{132} \mathrm{Cd}$ & 22.37 & ${ }^{138} \mathrm{Sn}$ & 26.74 & ${ }^{134} \mathrm{Cd}$ & 15.77 \\
${ }^{139} \mathrm{Sn}$ & 19.64 & ${ }^{134} \mathrm{Cd}$ & 25.96 & ${ }^{80} \mathrm{Ni}$ & 12.09 \\
${ }^{137} \mathrm{Sn}$ & 18.06 & ${ }^{137} \mathrm{Sn}$ & 23.23 & ${ }^{86} \mathrm{Zn}$ & 11.85 \\
${ }^{137} \mathrm{Sb}$ & 13.69 & ${ }^{140} \mathrm{Sn}$ & 21.79 & ${ }^{85} \mathrm{Zn}$ & 11.05 \\
${ }^{140} \mathrm{Sn}$ & 11.12 & ${ }^{86} \mathrm{Zn}$ & 21.15 & ${ }^{197} \mathrm{Hf}$ & 10.62 \\
${ }^{86} \mathrm{Zn}$ & 10.24 & ${ }^{139} \mathrm{Sn}$ & 17.25 & ${ }^{137} \mathrm{Sn}$ & 10.33 \\
${ }^{135} \mathrm{Sn}$ & 9.40 & ${ }^{136} \mathrm{Sn}$ & 16.61 & ${ }^{132} \mathrm{Cd}$ & 9.47 \\
${ }^{134} \mathrm{Cd}$ & 8.27 & ${ }^{133} \mathrm{Cd}$ & 14.33 & ${ }^{84} \mathrm{Zn}$ & 9.23 \\
${ }^{133} \mathrm{Cd}$ & 7.72 & ${ }^{135} \mathrm{Sb}$ & 13.80 & ${ }^{141} \mathrm{Sn}$ & 8.89 \\
${ }^{131} \mathrm{Cd}$ & 7.25 & ${ }^{131} \mathrm{Cd}$ & 13.16 & ${ }^{142} \mathrm{Sn}$ & 8.35 \\
${ }^{85} \mathrm{Zn}$ & 7.08 & ${ }^{141} \mathrm{Sb}$ & 12.25 & ${ }^{136} \mathrm{Cd}$ & 7.98 \\
${ }^{135} \mathrm{In}$ & 6.66 & ${ }^{133} \mathrm{In}$ & 12.04 & ${ }^{135} \mathrm{Cd}$ & 7.76 \\
${ }^{141} \mathrm{Sb}$ & 6.24 & ${ }^{85} \mathrm{Zn}$ & 11.92 & ${ }^{131} \mathrm{Cd}$ & 7.63 \\
${ }^{136} \mathrm{Sn}$ & 6.23 & ${ }^{135} \mathrm{Sn}$ & 11.54 & ${ }^{196} \mathrm{Lu}$ & 7.17 \\
${ }^{132} \mathrm{In}$ & 5.92 & ${ }^{133} \mathrm{Sn}$ & 11.52 & ${ }^{133} \mathrm{Cd}$ & 7.12 \\
${ }^{133} \mathrm{Sn}$ & 5.46 & ${ }^{139} \mathrm{Sb}$ & 10.77 & ${ }^{137} \mathrm{In}$ & 6.66 \\
${ }^{137} \mathrm{G} \mathrm{Ga}$ & 4.77 & ${ }^{135} \mathrm{In}$ & 10.72 & ${ }^{139} \mathrm{Sn}$ & 6.00 \\
${ }^{134} \mathrm{In} \mathrm{In}$ & 4.16 & ${ }^{137} \mathrm{In}$ & 8.14 & ${ }^{87} \mathrm{Ga}$ & 5.16 \\
${ }^{132} \mathrm{Sb}$ & 4.68 & ${ }^{137} \mathrm{Sb}$ & 9.72 & ${ }^{195} \mathrm{Yb}$ & 5.50 \\
${ }^{135} \mathrm{Sb}$ & 4.15 & ${ }^{134} \mathrm{Sb}$ & 7.61 & ${ }^{196} \mathrm{Yb}$ & 5.03 \\
\hline & 4.14 & ${ }^{134} \mathrm{Sn}$ & 7.50 & ${ }^{132} \mathrm{In}$ & 5.03 \\
\hline
\end{tabular}

world. Table 1 shows the top 25 nuclei with the greatest impact on the r-process for the three models. Since the particular isotopes of these elements that have the greatest impact can shift depending on the astrophysical conditions, a future paper will explore the effects of various astrophysical scenarios in determining the most important nuclei to measure.

This work was supported by the National Science Foundation through grant number PHY0758100 and the Joint Institute for Nuclear Astrophysics grant number PHY0822648.

Open Access This is an open access article distributed under the terms of the Creative Commons Attribution License (http://creativecommons.org/licenses/by/3.0), which permits unrestricted use, distribution, and reproduction in any medium, provided the original work is properly cited. 


\section{References}

1. C. Arlandini, F. Kappeler, K. Wisshak, Astrophys. J. 525, 886 (1999).

2. N. Grevesse, M. Asplund, A. Sauval, P. Scott, Astrophys. Space Sci. 328, 179 (2010).

3. K. Lodders, Astrophys. J. 591, 1220 (2003).

4. C. Sneden, J.J. Cowan, R. Gallino, Annu. Rev. Astron. Astrophys. 46, 241 (2008).

5. M. Arnould, S. Goriely, K. Takahashi, Phys. Rep. 450, 97 (2007).

6. K.-L. Kratz, J. Görres, B. Pfeiffer, M. Wiescher, J. Radioanal. Nucl. Chem. 243, 133 (2000).

7. S.E. Woosley, J.R. Wilson, G.J. Mathews, R.D. Hoffman, B.S. Meyer, Astrophys. J. 433, 229 (1994).

8. C. Freiburghaus, S. Rosswog, F.-K. Thielemann, Astrophys. J. 525, L121 (1999).

9. R. Surman, G.C. McLaughlin, W.R. Hix, Astrophys. J. 643, 1057 (2006).

10. R. Surman, S. Kane, J. Beun, G.C. McLaughlin, W.R. Hix, J. Phys. G 35, 014059 (2008).

11. S.-I. Fujimoto, K. Kotake, S. Yamada, M.-A. Hashimoto, K. Sato, Astrophys. J. 644, 1040 (2006).

12. S. Nishimura, K. Kotake, M.-A. Hashimoto, S. Yamada, N. Nishimura, S. Fujimoto, K. Sato, Astrophys. J. 642, 410 (2006).

13. W. Fowler, G. Caughlan, B. Zimmerman, Annu. Rev. Astron. Astrophys. 5, 525 (1967).

14. S. Wanajo, S. Goriely, M. Samyn, N. Itoh, Astrophys. J. 606, 1057 (2004).

15. K. Farouqi, K.-L. Kratz, B. Pfeiffer, T. Rauscher, F.-K. Thielemann, J.W. Truran, Astrophys. J. 712, 1359 (2010).
16. A. Arcones, G. Martinez-Pinedo, Phys. Rev. C 83, 045809 (2011).

17. H. Schatz, Phys. Today 61, 40 (2008).

18. J. Walsh, Ngam.f Fortran code, Clemson University (1994).

19. B.S. Meyer, Phys. Rev. Lett. 89, 231101 (2002).

20. Y.-Z. Qian, P. Vogel, G.J. Wasserburg, Astrophys. J. 494, 285 (1998)

21. Y.-Z. Qian, S.E. Woosley, Astrophys. J. 471, 331 (1996).

22. P. Möller, B. Pfeiffer, K.-L. Kratz, Phys. Rev. C 67, 055802 (2003).

23. T. Rauscher, F.-K. Thielemann, At. Data Nucl. Data Tables 75, 1 (2000).

24. G. Audi, A.H. Wapstra, C. Thibault, Nucl. Phys. A 729, 337 (2002).

25. P. Möller, J.R. Nix, W.D. Myers, W.J. Swiatecki, At. Data Nucl. Data Tables 59, 185 (1995).

26. J. Duflo, A.P. Zuker, Phys. Rev. C 52, R23 (1995).

27. S. Goriely, N. Chamel, J.M. Pearson, Phys. Rev. C 82, 035804 (2010).

28. G. Savard, R. Pardo, Proposal for the ${ }^{252}$ Cf source upgrade to the ATLAS facility, Technical report, ANL (2005).

29. O.B. Tarasov, M. Hausmann, LISE ++ development: Abrasion-Fission, Technical report, NSCL, MSUCL1300 (2005).

30. J.M. Pearson, R.C. Nayak, S. Goriely, Phys. Lett. B 387, 455 (1996).

31. A. Teymurazyan, A. Aprahamian, I.Bentley, N.Paul, in preparation (2012).

32. E.M. Burbidge, G.R. Burbidge, W.A. Fowler, F. Hoyle, Rev. Mod. Phys. 29, 547 (1957).

33. R. Surman, J. Beun, G.C. McLaughlin, W.R. Hix, Phys. Rev. C 79, 045809 (2009). 